\title{
Research on Vehicle Active Anti-Collision Warning System
}

\author{
Xie Fei ${ }^{1, a}$, Liu Wenying ${ }^{2, b}$ and Wang Jianhua ${ }^{1, c}$ \\ ${ }^{1}$ College of Automotive Engineering, Jilin University, Changchun, China \\ ${ }^{2}$ China Automotive Technology \& Research Center, Tianjin, China \\ axiefei620@126.com, ${ }^{b}$ liuwenying@catarc.ac.cn, ${ }^{\mathrm{c} I s d @ j l u . e d u . c n ~}$
}

Keywords: collision avoidance, target detection, safety distance, control strategy

Abstract. This paper discussed an automotive collision avoidance and warning system based on millimeter-wave radar. With the theory and application research of proactive early-warning and avoidance control, we have established the avoidance control strategy and algorithm. This system can identify dangerous targets and automatically determine whether the vehicle is in a dangerous condition. According to the level of risk status, it will take appropriate measures such as alarming, decelerating or even automatically braking to reduce the likelihood of rear-end accidents and improve driving safety on highway.

\section{Introduction}

The traffic accidents occur more frequently than ever. On highway, when some situations happen such as inclement weather happens, poor road conditions, traffic congestions or driver fatigue, the car would be prone to occur rear-end collisions, head-on collisions or even serial collision accidents. According to the statistics of traffic accidents, two-car rear-end collision accidents account for $65 \%$ and $80 \%$ of traffic accidents could be attributed to drivers' delayed response. Mercedes-Benz had done analysis of various types of accidents, and concluded that if the drivers could respond 0.5 seconds earlier they would avoid $60 \%$ of rear-end collisions. Besides, $40 \%$ of the side impacts and $30 \%$ of frontal collisions can also be prevented. If they could have more time in advance to take measures, the vast majority of traffic accidents would be effectively prevented[1][2]. Therefore, the vehicle active anti-collision warning system really has a great significance for the driving safety and it will achieve a broad market prospect.

\section{System Components}

Vehicle active anti-collision warning system belongs to the context of active safety technology, and it is an auxiliary device to help drivers automatically avoid a car collision [3]. When moving, the vehicle can automatically discover other cars, pedestrians or obstacles which may cause a dangerous threat to the vehicle within the field of vision. When the system has predicted the coming danger, it can remind the driver or automatically control the vehicle to avoid collision accidents.

Information Collection System. Information collection system consists of many sensors that used to obtain the host car speed, the target car speed, relative distance, road conditions and other information. Foremost among those sensors is the target detection device - radar, which now has three major categories widely used by us: ultrasound, optical and electromagnetic. The millimeter-wave radar is the most representative one within the electromagnetic category. The vehicle active anti-collision warning system described in this paper is based on millimeter-wave radar.

Data Processing System. After having received signals from target detection devices and vehicle sensors, the data processing system would analyze the collected date, and identify or classify the risk of targets. Then, according to the different levels of the dangerous conditions, the system would select the corresponding control strategies and let the appropriate actuators work.

Execution System. The execution system is used to receive control commands produced by the data processing system, then make appropriate actions to achieve the desired effect, and finally complete the corresponding collision avoidance action. Execution System includes the alarm device, 
deceleration mechanism and brake mechanism. The alarm equipment is installed on the operation panel. The deceleration function is achieved by the control of engine and gears. The brake mechanism includes brake system, ABS and ESP.

\section{Dangerous Targets Recognition And Avoidance Control Strategy}

Safety Distance Model. Based on the vehicle safety, the vehicle safety distance model can be established, which helps the driver keep safety distance between vehicles. This paper has analyzed the sports schemes of the front vehicle and the following vehicle when driving [4][5], and as it is shown in Fig. 1 . The speed of the following vehicle is $u_{1}$, and $u_{2}$ represents the front vehicle's speed. The safety distance between the head of the following vehicle and the rear of the front vehicle is $d$. After $t$ seconds, the speed of the following vehicle will be reduced to $u_{1}$, and cover the distance of $d_{1}$. Simultaneously, the speed of the front vehicle will be reduced to $u_{2}$, and cover $d_{2}$. At this time, the distance between the two vehicles will change to $d_{0}$, which represents the minimum safety distance that should be reserved to guarantee that the two vehicles are out of danger, and we usually set $d_{0}$ as $2 \sim 5 \mathrm{~m}$. The four distances presented above meet the following equation:

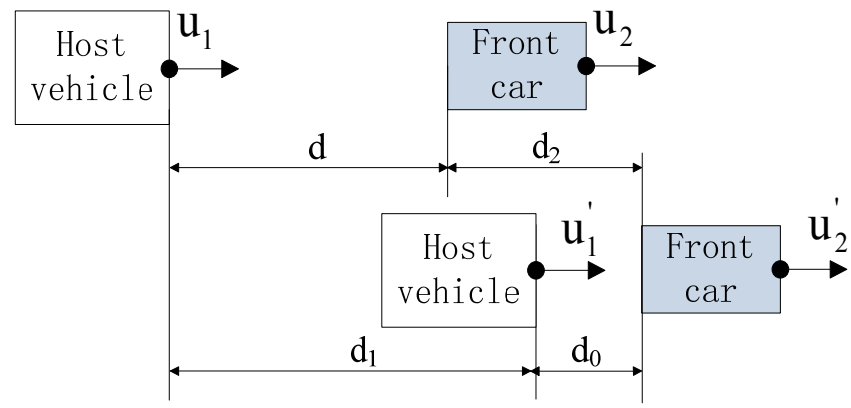

Fig.1 Moving vehicles spacing diagram

$d=d_{1}-d_{2}+d_{0}$

$d$ is used to represent the vehicle safety distance .It varies and means different critical distances in accordance with different situations. We can acquire four different distances according to the safety distance model: Lock-in Target distance Dt, Safety Critical distance Ds, Danger Critical distance Dd and Limited Critical distance DL. In this paper Drel and Vrel respectively represent the actual relative distance and relative speed between the target and the host vehicle.

Targets Recognition. The targets recognition principle is shown in Fig.2 and Fig.3. There appears four targets in the radar's scanning coverage except target F. In this situation, target D is the most dangerous one and we should set it as an important object of judgment. When target B goes across lane or target $C$ merges lane, there will be three targets that should be judged. According to the distance priority level, target $\mathrm{B}$ is most dangerous. Target $\mathrm{E}$ is not dangerous for its lane is far from the host vehicle's lane. From the above analyzing, we should set the targets which are driving in front of the host vehicle in the same lane as threatening targets and set the targets in the adjacent lane as potential threatening targets. The targets apart from the host vehicle's lane are non-threatening targets. The millimeter wave radar is located in the middle of the host vehicle. Usually the detection range of distance, angle and velocity respectively is $150 \mathrm{~m}, \pm 10^{\circ}$ and $\pm 150 \mathrm{~km} / \mathrm{h}$. The width of the lane is a and the width of the vehicle is $b$. In Fig. 3 if the corresponding parameter of target $B$ is $T_{b}\left(R_{b}, \theta_{b}, v_{r b}\right)$. 


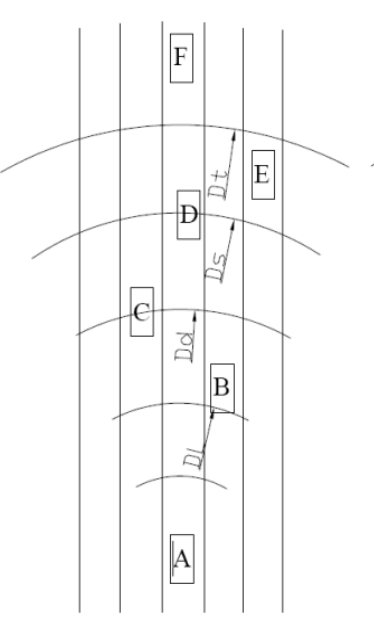

(a)

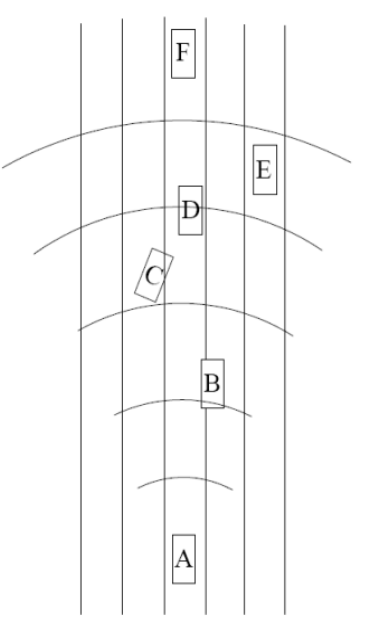

(b)

Fig.2 Vehicle driving environment simulation diagram

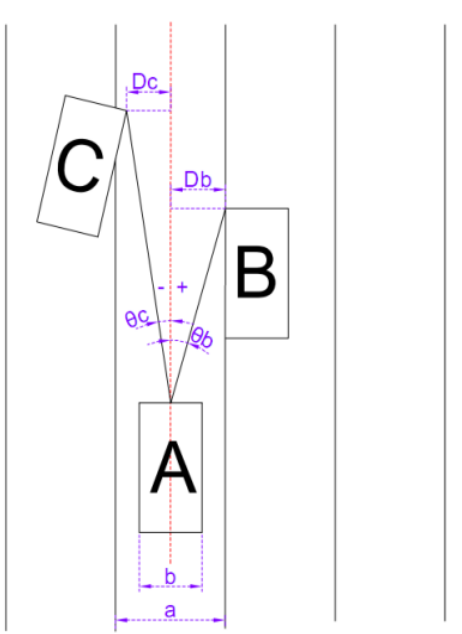

Fig.3 Targets recognition principle diagram

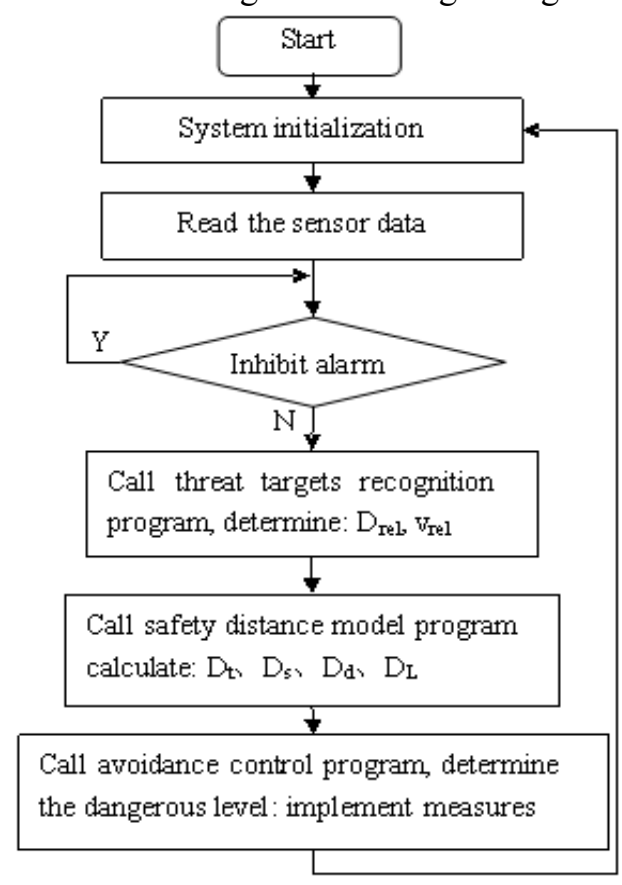

Fig.4 The main program flow chart

Avoidance Control Strategy. Two main control strategies are formulated according to the recognition of the dangerous targets and the difference of different drivers-tend-to-comfort and tend-to-safety.

When the real relative distance between the car and the threat targets detected by the millimeter wave radar is more than the Safety Critical Distance, the car is safe, and then the safety control strategy is implemented. When the relative distance is between the Safety Critical distance and the Danger Critical distance, the system would remind the drivers to drive carefully with low frequency warning sound of drops. This is alarm control strategy. When the distance of threat targets is less than the Danger Critical distance, the threat targets become dangerous ones. The system would remind the drivers to drive slowly with high frequency warning sound of drops and then the car will automatically slows down. This is Deceleration control strategy. When the relative distance of threat targets is less than the Limited Critical distance, the accident is unavoidable. The system would remind the drivers of emergency braking with high frequency warning sound of drops and then the car will automatically fast increase the braking force until the car is out of danger. This is brake control strategy. In Fig.4, $\mathrm{Dr}$ represents for the safe distance between vehicles prescribed by the highway. S0, S1, S2, S3 are the execution measures correspond to different judgments. They are corresponding to the four control strategies in proper sequence. 


\section{Simulation and Result Analysis}

By using MATLAB/Simulink we can set up the vehicle model, targets model, safety distance model and the avoidance controller model. The simulation analysis is used for several typical riding conditions. The simulation results are shown in Fig.5(a), (b) and (c) respectively represent for riding conditions of static targets, low-speed targets and deceleration targets.

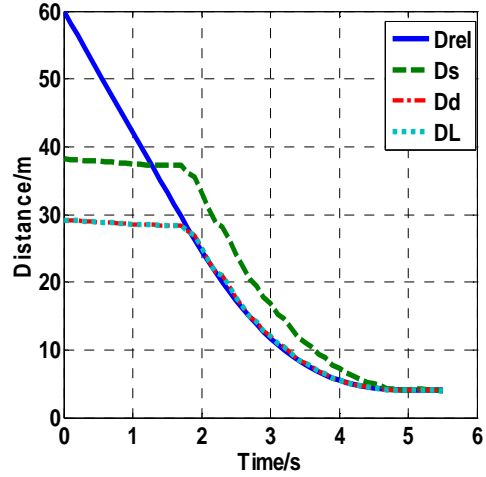

(a)

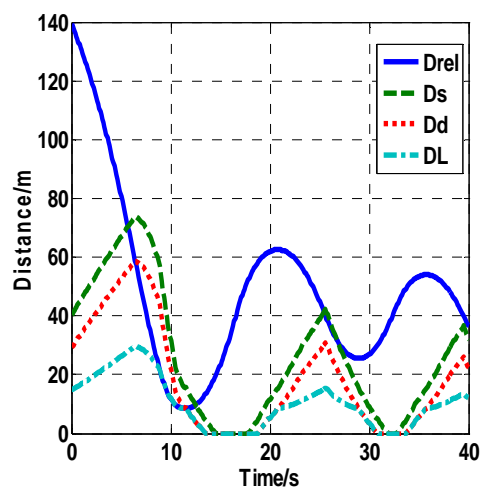

(b)

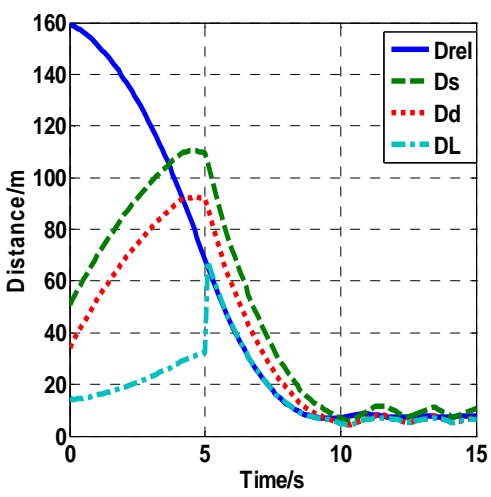

(c)

Fig.5 Simulation results of three conditions

During all the three working conditions, the drivers don't need to handle the vehicle. By comparing the results of actual distance Drel with $D s, D d$ and $D L$, we conclude that the actual distance is almost in the safe range, and is lower than safe distance only in some short time, but not lower than limited distance, so the host vehicle can get rid of the dangerous conditions and make sure the collision accident won't appear.

\section{Conclusion}

Considering the composition of the vehicle active anti-collision warning system, this paper focused on the study of avoidance control. It has formulated the dangerous targets recognition algorithm, and divided the targets into non-threatening targets, potential threatening targets and threatening targets. Two avoidance control strategies of tend-to-comfort and tend-to-safety have been put forward. According to the corresponding control strategy we have built the algorithm and flow. All of these have formed the foundation for the development of vehicle active anti-collision warning system. The model of the system has been set up and the results of simulation demonstrate that the control strategy is reasonable and the control algorithm is efficient. The system has good instantaneity and accuracy, and it can effectively avoid rear-end accidents on the highway. This study also has a certain instructive significance to develop the vehicle anti-collision system products.

\section{References}

[1] Wang C.D., Halajian S. Processing methods enhance collision-warning systems [J]. Microwaves \& RF, 1997, 36(3): 72-82.

[2] Woll J.D. VORAD collision warning radar[C].Radar Conference, Record of the IEEE, 1995 International, 1995

[3] Seiler P., Song B., Hedrick J.K. Development of a collision avoidance system [J]. Development, $1998,(4) 17-22$.

[4] WangJunLei, Research and development of the highway rear-end collision warning system [D].Chang-an university, 2005.

[5] Mclaughlin S.B. Analytic assessment of collision avoidance systems and driver dynamic performance in rear-end crashes and near-crashes [D]. Virginia Polytechnic Institute and State University, 2008. 